\title{
Flow of viscoelastic fluids between plates rotating about distinct axes
}

\author{
K. R. Rajagopal, M. Renardy, Y. Renardy, and A. S. Wineman \\ Department of Mechanical Engineering, University of Pittsburgh, \\ Department of Mathematics, Virginia Polytechnic Institute and State University, Blacksburg, and \\ Department of Mechanical Engineering and Applied Mechanics, University of Michigan, Ann Arbor
}

\begin{abstract}
We discuss the flow of BKZ fluids in an orthogonal rheometer. Some analytical results are proved, and numerical solutions are obtained for the Currie model. These solutions show a boundary layer behavior at high Reynolds numbers and the possibility of discontinuous solutions or nonexistence at high Weissenberg numbers.
\end{abstract}

Key words: Maxwell orthogonal rheometer, continuation method, Currie model, discontinuous solutions

\section{Introduction}

The flow in an orthogonal rheometer has been the object of considerable interest and has been studied by several authors (cf. Maxwell and Chartoff [1], Blyler and Kurtz [2], Bird and Harris [3], Huilgol [4], Kearsley [5], Gordon and Schowalter [6]). The apparatus consists of two parallel plates which rotate with the same constant angular velocity about distinct axes (see figure 1). In all these investigations the analysis was based on the assumption that inertial effects are negligible. Abbot and Walters [7] obtained an exact solution for the flow of a Newtonian fluid under the assumption that the plates are infinite. They also studied the flow of a viscoelastic fluid in such an apparatus, but in this case they assumed that the distance between the two axes of rotation is small and employed a power series expansion in the distance between the axes. This kind of expansion is also studied by Goldstein and Schowalter [8].

Rajagopal [9] showed that the equations of motion for an arbitrary simple fluid are compatible with the following ansatz for the velocity field:

$$
\begin{aligned}
& u=-\Omega[y-g(z)], \\
& v=\Omega[x-f(z)], \\
& w=0 .
\end{aligned}
$$

Here $u, v$ and $w$ are the $x, y$ and $z$ components of the velocity, and the plates of the rheometer are parallel to the $x, y$-plane. This velocity field describes a flow in 132 which each plane rotates rigidly with the same angular velocity $\Omega$. The locus of the centers of rotation is in general a curve in space defined by $x=f(z), y=g(z)$. The flow given by (1) is a motion with constant stretch history (cf. Coleman [10], Noll [11]). This implies that the equations of motion for a simple fluid reduce to the same order as the Navier-Stokes equations and that the no-slip boundary conditions are sufficient for determinacy. For related results, see also Goddard [12].

For certain special fluid models, the resulting boundary value problem can be solved exactly. Rajagopal and Gupta [13] obtained an exact solution for the incompressible fluid of second grade, while Rajagopal and Wineman [14] established exact solutions for a special class of BKZ fluid.

In this paper, we study the flow of a general BKZ fluid in the orthogonal rheometer. Detailed numerical results are provided for a specific model. We find that

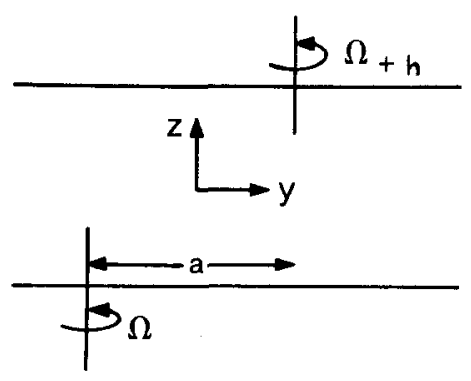

Fig. 1. Flow domain 
it is possible that the locus of the centers of rotation is piecewise continuously differentiable.

\section{Equations of motion}

The Cauchy stress $\boldsymbol{T}$ in an incompressible homogeneous simple fluid undergoing a motion with constant stretch history can be expressed as (cf. Wang [15])

$$
T=-p l+f\left(A_{1}, A_{2}, A_{3}\right),
$$

where $\boldsymbol{A}_{1}, \boldsymbol{A}_{2}$ and $\boldsymbol{A}_{3}$ denote the first three RivlinEricksen tensors (cf. Rivlin and Ericksen [16]) defined recursively by

$$
\boldsymbol{A}_{1}=(\nabla v)+(\nabla r)^{\mathrm{T}},
$$

and

$$
\boldsymbol{A}_{n}=\frac{d \boldsymbol{A}_{n-1}}{d t}+\boldsymbol{A}_{n-1}(\nabla v)+(\nabla v)^{\mathrm{\top}} \boldsymbol{A}_{n-1} .
$$

In these equations, $v$ denotes the velocity vector, $d / d t$ is the material time derivative and $\nabla v$ is defined according to the convention $(\nabla v)_{i j}=\partial v_{i} / \partial x_{j}$.

For the motion under consideration, it can be shown that (cf. Rajagopal [9])

$$
\boldsymbol{A}_{3}=-\Omega^{2} \boldsymbol{A}_{1} \text {, }
$$

and hence (2) reduces to ${ }^{1}$ )

$$
\boldsymbol{T}=-p \mathbf{1}+\boldsymbol{f}\left(\boldsymbol{A}_{1}, \boldsymbol{A}_{2}\right) \text {. }
$$

Since $A_{1}$ and $A_{2}$ depend only on $z$, the equation of motion reduces to

$$
\begin{aligned}
& \frac{d f_{13}}{d z} \boldsymbol{i}+\frac{d f_{23}}{d z} \boldsymbol{j}+\frac{d f_{33}}{d z} \boldsymbol{k} \\
& =\nabla p+\nabla(\varrho \Phi)-\varrho \Omega^{2}[x-f(z)] \boldsymbol{i}-\varrho \Omega^{2}[y-g(z)] \boldsymbol{j},
\end{aligned}
$$

where $\boldsymbol{i}, \boldsymbol{j}$ und $\boldsymbol{k}$ denote unit vectors in the $x, y$ and $z$ directions, respectively. In obtaining (6) we have also assumed that the body force is conservative and derivable from a potential $\Phi$. By operating on (6) with the curl operator, we find

$$
\begin{aligned}
& \frac{d^{2} f_{13}}{d z^{2}}=\frac{d}{d z}\left\{\varrho \Omega^{2} f(z)\right\}, \\
& \frac{d^{2} f_{23}}{d z^{2}}=\frac{d}{d z}\left\{\varrho \Omega^{2} g(z)\right\} .
\end{aligned}
$$

1) The same form is obtained for viscometric flows where $\boldsymbol{A}_{3}=\mathbf{0}$. However, the function $\boldsymbol{f}$ appearing in (5) is in general different from that in viscometric flow.
These equations can be integrated to yield

$$
\begin{aligned}
& \frac{d f_{13}}{d z}=\varrho \Omega^{2} f(z)+s, \\
& \frac{d f_{23}}{d z}=\varrho \Omega^{2} g(z)+q,
\end{aligned}
$$

where $q$ and $s$ are constants. If $f$ and $g$ satisfy eqs. (8), then (6) is satisfied if and only if the pressure is given by

$p=-\varrho \Phi+\frac{\varrho \Omega^{2}}{2}\left(x^{2}+y^{2}\right)+s x+q y+f_{33}(z)+C$,

where $C$ is an arbitrary constant. Hence the system (8) can be regarded as equivalent to (6).

Since the fluid adheres to the rotating plates, the appropriate boundary conditions for the problem are (see figure 1)

$$
\begin{aligned}
& u=\frac{\Omega a}{2}-\Omega y, v=\Omega x, w=0 \text { at } z=h, \\
& u=-\frac{\Omega a}{2}-\Omega y, v=\Omega x, w=0 \quad \text { at } \quad z=0 .
\end{aligned}
$$

When inserted into (1), these conditions lead to

$$
f(0)=f(h)=0, g(0)=-a / 2, g(h)=a / 2 \text {. }
$$

For the following, we consider fluids in which the Cauchy stress tensor $\boldsymbol{T}$ is related to the history of the deformation in the following manner (cf. Bernstein, Kearsley and Zapas [17])

$$
\boldsymbol{T}=-p \mathbf{1}+2 \int_{-\infty}^{t}\left\{U_{1} \boldsymbol{C}_{t}^{-1}(\tau)-U_{2} C_{l}(\tau)\right\} d \tau
$$

Here $C_{t}$ is the right relative stretch tensor defined by

$$
\boldsymbol{C}_{t}(\tau)=\boldsymbol{F}_{t}^{\top}(\tau) \boldsymbol{F}_{t}(\tau),
$$

and $U_{i}$ is the derivative of the stored energy function $U=U\left(I_{1}, I_{2}, t-\tau\right)$ with respect to the argument $I_{i}$. The latter are defined by

$$
I_{1}=\operatorname{tr} C_{t}^{-1}(\tau), \quad I_{2}=\operatorname{tr} C_{t}(\tau) .
$$

It follows from a lengthy but straightforward computation that the equation of motion reduces to

$$
\begin{aligned}
& \frac{d}{d z}\left\{f^{\prime}(z) B(x)+g^{\prime}(z) A(x)\right\}=\varrho \Omega^{2} f(z)+s, \\
& \frac{d}{d z}\left\{-f^{\prime}(z) A(x)+g^{\prime}(z) B(x)\right\}=\varrho \Omega^{2} g(z)+q .
\end{aligned}
$$

The functions $A$ and $B$ are defined by

$$
A(x)=2 \int_{0}^{\infty} \tilde{U}[3+2(1-\cos \Omega \alpha) \varkappa, \alpha] \sin \Omega \alpha d \alpha,
$$




$$
B(x)=2 \int_{0}^{x} \tilde{U}[3+2(1-\cos \Omega \alpha) x, \alpha](1-\cos \Omega \alpha) d x,
$$

where

$$
\tilde{U}[I, \alpha]=U_{1}(I, I, \alpha)+U_{2}(I, I, \alpha) .
$$

The quantity $x$ is defined as

$$
x(z)=\left[f^{\prime}(z)\right]^{2}+\left[g^{\prime}(z)\right]^{2} .
$$

In this work, we shall find numerical solutions of the system (15) with boundary conditions (11) for a special class of BKZ fluids. Before we do this, however, we first remark on a few general characteristics of the problem under consideration.

\section{Analysis}

The solution to the system (15) depends on the material properties of the model. However, when $a=q=s=0$, we always have the trivial solution $f=g=0$. If $a, q$ and $s$ are small, this trivial solution can be uniquely continued to a solution $f(z ; a, q, s)$ and $g(z ; a, q, s)$. As the values of $a, q$ and $s$ are further increased, the process of unique continuation can only fail if one of the following situations occurs:

(i) the equations become singular,

(ii) the solution and/or its derivative become infinite,

(iii) the linearization of (15) at $f(z ; a, q, s), g(z ; a, q, s)$ has a nontrivial solution.

Whether any of these situations obtains depends on the choice of the constitutive equation which determines the functions $A$ and $B$. In the following, we discuss some implications of each of these conditions.

\section{Case (i)}

Eqs. (15) imply that

$$
\begin{aligned}
& f^{\prime \prime}\left[B+2 B^{\prime} f^{\prime 2}+2 A^{\prime} f^{\prime} g^{\prime}\right] \\
& +g^{\prime \prime}\left[A+2 B^{\prime} f^{\prime} g^{\prime}+2 A^{\prime} g^{\prime 2}\right]=\varrho \Omega^{2} f+s, \\
& f^{\prime \prime}\left[-A-2 A^{\prime} f^{\prime 2}+2 B^{\prime} f^{\prime} g^{\prime}\right] \\
& +g^{\prime \prime}\left[B-2 A^{\prime} f^{\prime} g^{\prime}+2 B^{\prime} g^{\prime 2}\right]=\varrho \Omega^{2} g+q .
\end{aligned}
$$

This system becomes singular if and only if the determinant of the coefficients of the second derivatives becomes zero. This determinant is given by

$D=A^{2}+B^{2}+2 \varkappa\left(A A^{\prime}+B B^{\prime}\right)=\frac{d}{d \varkappa}\left\{\varkappa\left(A^{2}+B^{2}\right)\right\}$.

If the function $x \rightarrow x\left(A^{2}+B^{2}\right)$ is strictly monotonic, then $D$ can never be zero. This is the case for the models studied by Rajagopal and Gupta [13] and Rajagopal and Wineman [14] where $A$ and $B$ are constant.

\section{Case (ii)}

We now assume that the system (15) is non-singular, and discuss some of the implication of (ii). The following lemma shows that if $D$ and $B$ are positive, and the derivative of $f$ or $g$ becomes infinite, then this must happen at the boundary.

Lemma: If $D$ and $B$ are positive, then $x$ cannot have an interior maximum.

Proof: If we differentiate eqs. (15) and evaluate at $\varkappa^{\prime}=0$, we find that

$$
\begin{aligned}
& f^{\prime \prime \prime} B+g^{\prime \prime \prime} A+f^{\prime} B^{\prime} x^{\prime \prime}+g^{\prime} A^{\prime} x^{\prime \prime}=\varrho \Omega^{2} f^{\prime}, \\
& -f^{\prime \prime \prime} A+g^{\prime \prime \prime} B-f^{\prime} A^{\prime} x^{\prime \prime}+g^{\prime} B^{\prime} x^{\prime \prime}=\varrho \Omega^{2} g^{\prime} .
\end{aligned}
$$

Multiplying (21) by $f^{\prime} B+g^{\prime} A$ and $(21)_{2}$ by $g^{\prime} B-f^{\prime} A$, and adding the two, we obtain

$$
\begin{aligned}
& \left(A^{2}+B^{2}\right)\left(f^{\prime \prime \prime} f^{\prime}+g^{\prime \prime \prime} g^{\prime}\right)+\left(A A^{\prime}+B B^{\prime}\right) \varkappa \chi^{\prime \prime} \\
& =\varrho \Omega^{2} \varkappa B .
\end{aligned}
$$

It follows from the definition (18) of $x$ and from (20) that (22) can be rewritten as

$$
\frac{1}{2} D \varkappa^{\prime \prime}=\left(A^{2}+B^{2}\right)\left(f^{\prime \prime 2}+g^{\prime \prime 2}\right)+\varrho \Omega^{2} \chi B .
$$

Since $B$ and $D$ are postive, it follows that $x^{\prime \prime}>0$. Hence any interior point where $x^{\prime}=0$ is a minimum.

If further assumptions are added, it is possible to say more.

Lemma: If $A$ and $B$ are positive, bounded functions of $\varkappa$ and they have strictly positive lower bounds, then cuse (ii) does not happen.

Proof: We multiply $(15)_{1}$ by $f,(15)_{2}$ by $g$, add them and integrate. This leads to

$$
\begin{gathered}
\int_{0}^{h} g \Omega^{2}\left(f^{2}+g^{2}\right)+B\left(f^{\prime 2}+g^{\prime 2}\right)+s f+q g d z \\
=a / 2\left[-f^{\prime}(h) A(\varkappa(h))+g^{\prime}(h) B(\varkappa(h))\right. \\
\left.-f^{\prime}(0) A(\varkappa(0))+g^{\prime}(0) B(\varkappa(0))\right] .
\end{gathered}
$$

Next, we multiply $(15)_{1}$ by $g$ and $(15)_{2}$ by $f$, subtract them and integrate. This leads to

$$
\begin{aligned}
& \int_{0}^{h} A\left(f^{\prime 2}+g^{\prime 2}\right)+s g-q f d z \\
& =a / 2\left[f^{\prime}(h) B(\varkappa(h))+g^{\prime}(h) A(\varkappa(h))\right. \\
& \left.\quad+f^{\prime}(0) B(\varkappa(0))+g^{\prime}(0) A(\varkappa(0))\right] .
\end{aligned}
$$

It follows easily that the $H^{1}$-norm of $f$ and $g$ can be estimated in terms of the boundary terms occurring on 
the right side of either (24) or (25). If the $H^{1}$-norm stays bounded while following a branch of solutions, then the minima of $\left|f^{\prime} B+g^{\prime} A\right|$ and $\left|-f^{\prime} A+g^{\prime} B\right|$ also stay bounded, and so do the maxima of $|f|$ and $g \mid$. By integrating (15) with respect to $z$, it follows that $\left|f^{\prime} B+g^{\prime} A\right|$ and $\left|-f^{\prime} A+g^{\prime} B\right|$ remain uniformly bounded, and the assumptions on $A$ and $B$ imply that $f^{\prime}$ and $\left|g^{\prime}\right|$ also remain uniformly bounded. In order for case (ii) to happen, it is therefore necessary that the terms on the right of (24) and (25) both tend to infinity. Again by integrating (15) with respect to $z$, we find that this is possible only if the minima of $f^{\prime} B+g^{\prime} A$ and $-f^{\prime} A+g^{\prime} B$ both tend to $+\infty$. In this case, however, we conclude that

$$
g^{\prime}=\frac{A}{A^{2}+B^{2}}\left(f^{\prime} B+g^{\prime} A\right)+\frac{B}{A^{2}+B^{2}}\left(-f^{\prime} A+g^{\prime} B\right)
$$

tends to $+\infty$ uniformly on $[0, h]$, which is inconsistent with the boundary conditions.

Whether case (ii) ever obtains depends of course on the model under consideration. In the models considered by Rajagopal and Gupta [13] and Rajagopal and Wineman [14], the above lemma shows that case (ii) cannot obtain.

\section{Case (iii)}

Depending on the model under consideration, this case could lead to limit points or bifurcations. This cannot happen as long as the matrix of the coefficients of $f^{\prime \prime}$ and $g^{\prime \prime}$ in equations (19) remains positive definite. This is the case as long as

$$
B>0 \text { and } B^{2}+2 B B^{\prime} \chi-A^{\prime 2} \chi^{2}>0 .
$$

If $A$ and $B$ are positive constants, such as in [13] and [14], (27) holds ${ }^{2}$ ).

In summary, we found that neither of the cases (i) - (iii) ever occurs in a simple model where $A$ and $B$ are positive constants. Below, we report numerical results for a model where $A$ and $B$ are rather complicated expressions. The results clearly indicate that case (i) occurs in this model.

\section{Numerical solutions}

Let the interval $[0, h]$ be partitioned into $N$ equal subintervals bounded by points $z_{i}=(i-1) h / N, i=1,2, \ldots, N+1$. Define the notation $f_{i}=f\left(z_{i}\right), g_{i}=g\left(z_{i}\right)$. The finite difference

$\left.{ }^{2}\right)$ Of course this analysis only shows the absence of bifurcations within the class of solutions described by the kinematics of eq. (1). There may well be other possible flows which cannot be described by (1).

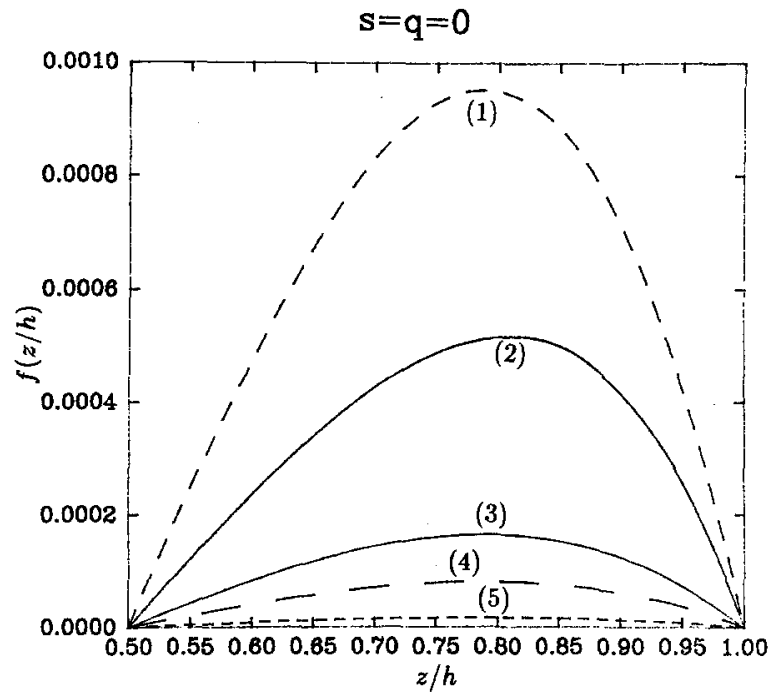

Fig. 2. Graph of $f(z / h)$ versus $z / h, s=q=0$.

$\lambda=0.5, C=2.5, \varrho=1, h=2$, zero-shear-rate viscosity $=10$.

(1) $a=1.0, R e=0.2, W e=1.0$.

(2) $a=0.1, R e=2.0, W e=10.0$.

(3) $a=1.0, R e=0.04, W e=0.2$.

(4) $a=0.1, R e=0.2, W e=1.0$.

(5) $a=0.1, R e=0.04, W e=0.2$

scheme used to approximate the system (15) is given by

$$
\begin{aligned}
& \Delta^{+}\left[\Delta^{-} f B\left(x_{-}\right)+\Delta^{-} g A\left(x_{-}\right)\right]=\varrho \Omega^{2} f_{i}+s, \\
& \Delta^{+}\left[-\Delta^{-} f A\left(x_{-}\right)+\Delta^{-} g B\left(x_{-}\right)\right]=\varrho \Omega^{2} g_{i}+q,
\end{aligned}
$$

where, with $\Delta h=h / N$,

$$
\Delta^{-}(.)=\frac{(.)_{i}-(.)_{i-1}}{\Delta h}, \Delta^{+}(.)=\frac{(.)_{i+1}-(.)_{i}}{\Delta h},
$$

and

$$
x_{-}=\left(\Delta^{-} f\right)^{2}+\left(\Delta^{-} g\right)^{2} .
$$

Since by (11), $f_{1}=f_{N+1}=0$ and $g_{1}=-a / 2, g_{N+1}=a / 2$, there are $2 N-2$ unknowns, $f_{2}, \ldots, f_{N}$ and $g_{2}, \ldots, g_{N}$. This discretization leads to the following nonlinear equations

$$
\begin{aligned}
& \left(f_{i+1}-f_{i}\right) B\left(x_{+}\right)+\left(g_{i+1}-g_{i}\right) A\left(x_{+}\right)-\left(f_{i}-f_{i-1}\right) B\left(x_{-}\right) \\
& -\left(g_{i}-g_{i-1}\right) A\left(x_{-}\right)-(\Delta h)^{2}\left(\varrho \Omega^{2} f_{i}+s\right)=0, \\
& -\left(f_{i+1}-f_{i}\right) A\left(x_{+}\right)+\left(g_{i+1}-g_{i}\right) B\left(x_{+}\right)+\left(f_{i}-f_{i-1}\right) A\left(x_{-}\right) \\
& -\left(g_{i}-g_{i-1}\right) B\left(x_{-}\right)-(\Delta h)^{2}\left(\varrho \Omega^{2} g_{i}+q\right)=0
\end{aligned}
$$

for each mesh interval point $z_{i}, i=2, \ldots, N$.

Denote eqs. (31) 1 and $(31)_{2}$ respectively by

$$
\begin{aligned}
& E_{1}\left(f_{i-1}, g_{i-1}, f_{i}, g_{i}, f_{i+1}, g_{i+1}\right)=E_{1}^{(i)}=0, \\
& E_{2}\left(f_{i-1}, g_{i-1}, f_{i}, g_{i}, f_{i+1}, g_{i+1}\right)=E_{2}^{(i)}=0, \\
& \quad i=2, \ldots, N .
\end{aligned}
$$

This system is solved using Newton's scheme on the vector equation $V=0$ where

$$
V=\left(\begin{array}{c}
E_{1}^{(2)} \\
E_{2}^{(2)} \\
\vdots \\
E_{1}^{(N)} \\
E_{2}^{(N)}
\end{array}\right) .
$$


The corresponding Jacobian is $(2 N-2)$ square and is banded with three sub-diagonals above and below the main diagonal:

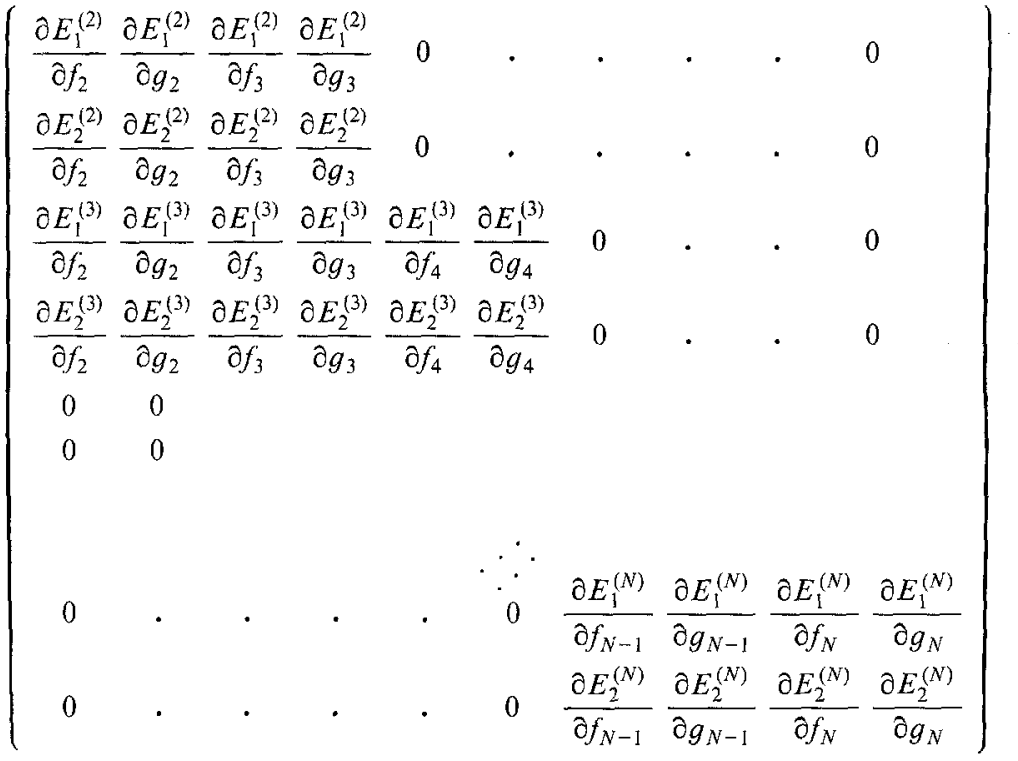

Several tests were carried out for the convergence of Newton's iteration scheme. The first uses the exact solution obtained by Rajagopal and Wineman [14] for the case in which $A(x)$ and $B(x)$ are constants and $s=q=0$. Let $f_{i}^{(e)}$ and $g_{i}^{(e)}$ denote the values of $f(z)$ and $g(z)$ given by the exact solution in [14] and let $f_{i}^{(c)}, g_{i}^{(c)}$ denote the values computed from Newton's iteration. Calculations were carried out for $\varrho=3, \Omega=1, a=0.1, h=2, B(x)=3, A(x)=2$ and a Newton's scheme tolerance of $10^{-5}$. The absolute errors $f_{i}^{(e)}-f_{i}^{(c)}$, $g_{i}^{(e)}-g_{i}^{(c)}$ were evaluated as the number of points was doubled from $N=10$ to $N=20$ and doubled again to $N=40$. The errors went down by $1 / 4$ each time indicating quadratic convergence as would theoretically be expected.

Next, $A(x)$ and $B(x)$ were prescribed to be

$$
\begin{aligned}
& A(x)=a_{1} x+a_{2}, \\
& B(x)=b_{1} x+b_{2},
\end{aligned}
$$

with $a_{1}, a_{2}, b_{1}, b_{2}$ prescribed constants. An exact solution for $f(z)$ and $g(z)$ is unknown for this case. In order to test the convergence of the Newton iteration scheme, the problem was modified in the following manner. $f(z)$ and $g(z)$ were chosen in the form

$$
\begin{aligned}
& f(z)=f_{1}\left[(z-h / 2)^{2}-(h / 2)^{2}\right], \\
& g(z)=g_{1}(z-h / 2)^{3}+g_{2}(z-h / 2),
\end{aligned}
$$

where $f_{1}, g_{1}, g_{2}$ are prescribed constants. Note that in accordance with $(11), f(0)=f(h)=0$ and $g(0)=-g(h)$. The $f$ and $g$ as given by (36) do not satisfy equations (15). We can, however, make them satisfy the equations by adding a forcing term to the right hand side of (15). When discretizing, we obtain a corresponding forcing term to be added to the right side of (32). We denote these terms by $\bar{E}_{1}^{(i)}, \bar{E}_{2}^{(i)}$.

The original system (32) is thus modified to

$$
\begin{aligned}
& E_{1}^{(i)}=\bar{E}_{1}^{(i)}, \\
& E_{2}^{(i)}=\bar{E}_{2}^{(i)}, \quad i=2, \ldots, N,
\end{aligned}
$$

for which (36) 1 and (36) 2 provide the exact solution. Computations were carried out for the two sets of parameters: $\left(\varrho, \Omega, h, a_{1}, a_{2}, b_{1}, b_{2}, f_{1}, g_{1}, g_{2}\right)=(3,1,2,1,1,1,1,1,1,1)$, $(3,1,2,1.5,2.9,3.1,10.0,0.1,2.4,5.2)$ with $10,20,40$ and 80 meshpoints. Comparison of the computed values and its exact solutions showed quadratic convergence.

In the final case, the process was repeated for

$$
\begin{aligned}
& A(x)=a_{1} x+a_{2}+a_{3} x^{2}, \\
& B(x)=b_{1} x+b_{2}+b_{3} x^{3} .
\end{aligned}
$$

Computations were performed for the second choice of parameters above, with $a_{3}=1, b_{3}=0$ and with $a_{3}=1, b_{3}=1$. The results again showed quadratic convergence.

\section{The Currie model}

Computations were carried out using the form of the stored energy function $U$ derived by Currie [18] as an approximation for the Doi-Edwards model (cf. [19]). It is given by

$$
U\left(I_{1}, I_{2}, s\right)=m(s) \hat{U}(J),
$$

where

$$
\begin{aligned}
& \hat{U}(J)=5 \ln (J-1)-9.73, \\
& J=I_{1}+2\left(I_{2}+3.25\right)^{1 / 2},
\end{aligned}
$$

and

$$
m(s)=-\dot{G}(s) .
$$

$G(s)$ is the stress relaxation function of linear viscoelasticity. For the present calculations,

$$
m(s)^{\prime}=C e^{-\lambda s} \text {. }
$$


The form of the function $\tilde{U}(I, \alpha)$ defined in (17) is

$$
\tilde{U}(I, \alpha)=C e^{-\lambda \alpha} \bar{U}(I),
$$

where

$$
\bar{U}(I)=\frac{5}{I-1+2 \sqrt{I+3.25}}\left(1+\frac{1}{\sqrt{I+3.25}}\right) .
$$

Functions $A$ and $B$ defined through (16) become

$$
\begin{aligned}
& A=2 C \int_{0}^{\infty} e^{-\lambda \alpha} \bar{U}[3+2(1-\cos \Omega \alpha) x] \sin \Omega \alpha d \alpha, \\
& B=2 C \int_{0}^{\infty} e^{-\lambda \alpha} \bar{U}[3+2(1-\cos \Omega \alpha) x](1-\cos \Omega \alpha) d \alpha .
\end{aligned}
$$

For convenience in numerical integrations, these integrals can be re-expressed as

$$
\begin{aligned}
A= & \frac{2 C}{\Omega[1-\exp (-2 \pi \lambda / \Omega)]} \\
& \cdot \int_{0}^{2 \pi} e^{-\lambda \alpha^{\prime} / \Omega} \bar{U}\left[3+2\left(1-\cos \alpha^{\prime}\right) \varkappa\right] \sin \alpha^{\prime} d \alpha^{\prime}, \\
B= & \frac{2 C}{\Omega[1-\exp (-2 \pi \lambda / \Omega)]} \\
& \cdot \int_{0}^{2 \pi} e^{-\lambda \alpha^{\prime} / \Omega} \bar{U}\left[3+2\left(1-\cos \alpha^{\prime}\right) x\right]\left(1-\cos \alpha^{\prime}\right) d \alpha^{\prime},
\end{aligned}
$$

where the non-dimensional time $\alpha^{\prime}=\alpha \Omega$ has been introduced.

The parameters of the problem are now: $a, h$ with dimension $L ; \Omega, \lambda$ with dimension $T^{-1} ; \varrho$ with dimension $M / L^{3}$; and $C$ with dimension $M /\left(L T^{3}\right)$. Note $C / \lambda^{2}$ is the zero shear rate viscosity. From these the following non-dimensional parameters are formed; $a / h$, a Weissenberg number $W e=\Omega / \lambda$ and a Reynolds number $R e=g h^{2} \Omega \lambda^{2} / C$. These appear in the nondimensionalized version of eqs. (15)

$$
\begin{aligned}
& \frac{d}{d \bar{z}}\left(\bar{B} \frac{d \bar{f}}{d \bar{z}}+\bar{A} \frac{d \bar{g}}{d \bar{z}}\right)=\operatorname{ReWe} \bar{f}+\bar{s}, \\
& \frac{d}{d \bar{z}}\left(-\bar{A} \frac{d \bar{f}}{d \bar{z}}+\bar{B} \frac{d \bar{g}}{d \bar{z}}\right)=\operatorname{ReWe} \bar{g}+\bar{q},
\end{aligned}
$$

where $\bar{f}=f / a, \quad \bar{g}=g / a, \quad \bar{z}=z / h, \quad \bar{A}=A \lambda / C \quad$ and $\bar{B}=B \lambda / C$.

The Jacobian associated with Newton's method requires the evaluation of the derivatives $d A / d x$ and $d B / d x$. Expressions for these are given by

$$
\begin{aligned}
& \frac{d A}{d x}=\frac{4 C}{\Omega[1-\exp (-2 \pi \lambda / \Omega)]} \\
& \cdot \int_{0}^{2 \pi} e^{-\lambda \alpha^{\prime} / \Omega} \bar{U}^{\prime}\left[3+2\left(1-\cos \alpha^{\prime}\right) \chi\right]\left(1-\cos \alpha^{\prime}\right) \sin \alpha^{\prime} d \alpha^{\prime}
\end{aligned}
$$

$$
\begin{aligned}
& \frac{d B}{d x}=\frac{4 C}{\Omega[1-\exp (-2 \pi \lambda / \Omega)]} \\
& \cdot \int_{0}^{2 \pi} e^{-\lambda \alpha^{\prime} / \Omega} \bar{U}^{\prime}\left[3+2\left(1-\cos \alpha^{\prime}\right) \chi\right]\left(1-\cos \alpha^{\prime}\right)^{2} d \alpha^{\prime} .
\end{aligned}
$$

Each of the integrals in (47) and (49) is evaluated by subdividing the interval $[0,2 \pi]$ into subintervals of equal length and then using the trapezoidal rule.

The following are the components of traction at the walls $z=0, h$ which were also computed:

$$
\begin{aligned}
& t_{x}=B(\varkappa, \Omega) f^{\prime}+A(\varkappa, \Omega) g^{\prime}, \\
& t_{y}=-A(\varkappa, \Omega) f^{\prime}+B(\varkappa, \Omega) g^{\prime} .
\end{aligned}
$$

By comparing this with eqs. (15), we find that if $s h+\varrho \Omega^{2} \int_{0}^{h} f(z) d z$ is not zero, then the component of traction $t_{x}$ has different values at the top and bottom plates. Similarly, if $q h+\varrho \Omega^{2} \int_{0}^{h} g(z) d z$ is not zero, then the component of traction $t_{y}$ would be different at the top and bottom plates. This was also checked numerically for the figures discussed below.

\section{Results and discussion}

The three non-dimensional parameters governing the problem are the Reynolds and the Weissenberg numbers and $a / h$. Rajagopal and Wineman [14] restricted themselves to the special case when $s=q=0$. In the case of small Reynolds numbers, they found their solutions for $f$ and $g$ to be approximately linear. However, at high Reynolds numbers, they found a boundary layer structure in their solution. The model considered here is much more complicated than that considered in [14] and it is possible that the solutions possess a more complicated structure.

We shall first discuss the situation when the Reynolds number is small and the Weissenberg number is large. When $s=q=0$, we find that, below some critical value of $a / h$, the numerical scheme converges to a solution which is anti-symmetric about the mid-plane with $f \approx 0$ and $g$ being approximately linear as indicated in figures $2-5$. (Note that $f=0$ and $g$ linear is an exact solution for $R e=0$.) Figure 2 shows that $f \sim \Omega$ for small $\Omega$ and $f \sim a$ for small $a$. Figure 3 shows that $g(z) \sim a(z / h-0.5)$ for small $\Omega$ and $a$. At $W e=10$, $R e=2$, Newton's scheme converged quickly until $a$ reached approximately 0.94 , above which it diverged. As the critical value of $a$ is approached, the discriminant $D \equiv A^{2}+B^{2}+2 \varkappa\left(A A^{\prime}+B B^{\prime}\right)$ approaches zero 


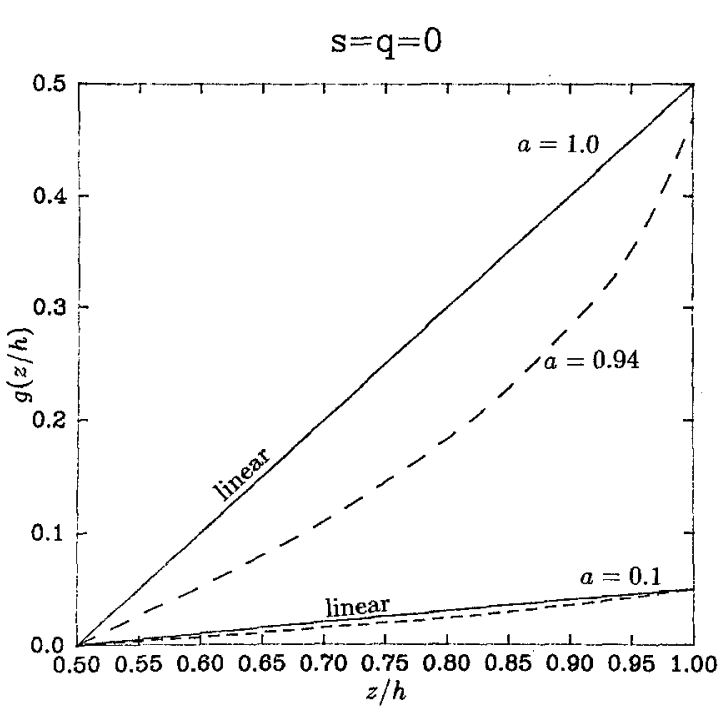

Fig. 3. Graph of $g(z / h)$ versus $z / h, s=q=0$.

$\lambda=0.5, C=2.5, \varrho=1, h=2$, zero-shear-rate viscosity $=10$.

$\ldots: R e=0.2, W e=1.0$ and $R e=0.04, W e=0.2$.

$\cdots: R e=2.0, W e=10.0$

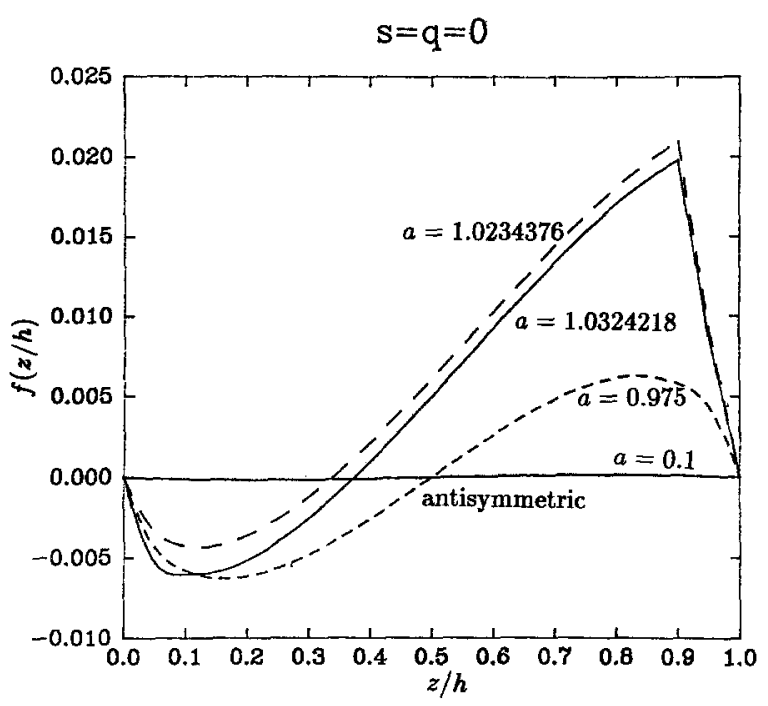

Fig. 4. Graph of $f(z / h)$ versus $z / h, s=q=0$.

$\lambda=1, C=1, \varrho=0.1, h=1, \Omega=4, W e=4, R e=0.4$, zeroshear-rate viscosity $=1$. Twenty meshpoints were used in the finite-difference scheme

and the Newton scheme has convergence problems. The solutions shown have been tested for convergence with 20 and 40 meshpoints. Figures 4 and 5 display solutions with discontinuous derivatives when $W e=4$, $R e=0.4$. The solutions possess the anti-symmetric structure for $a$ up to about 0.9750 , above which the discriminant $D$ approaches zero. For larger values of $a$, solutions are not unique. They may possess discontinuous slopes with the discriminant $D$ becoming nega-

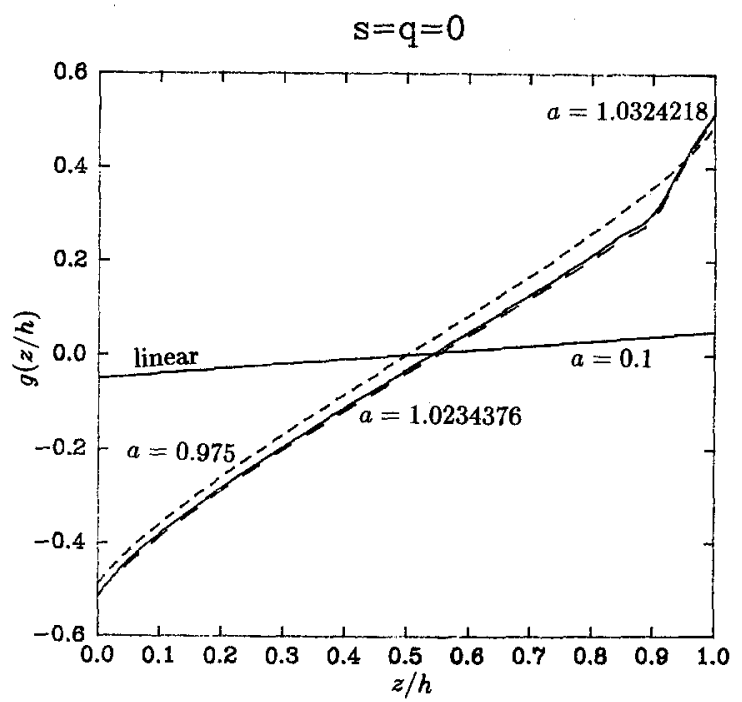

Fig. 5. Graph of $g(z / h)$ versus $z / h, s=q=0$.

$\lambda=1, C=1, \varrho=0.1, h=1, \Omega=4, W e=4, R e=0.4$, zeroshear-rate viscosity $=1$. Twenty meshpoints were used in the finite-difference scheme

tive on part of the solution. Which solution the Newton iteration would converge to depends on, e.g., the number of meshpoints. We display the results obtained with 20 meshpoints for $a$ above 0.9750 . It is found that the discontinuous solutions are not anti-symmetric. This breakdown in symmetry is purely due to the round-off error in the numerical scheme rather than any non-symmetric input into the problem. In view of experiments, it is interesting to note that the tractions on the plates can be different for these discontinuous solutions. It is evident from figure 4 that the integral of $f$ is not zero, and hence $t_{x}$ must have different values at the two plates. Direct computation of the tractions showed that both $t_{x}$ and $t_{y}$ have different values on the plates. Note that there is no difference in the tractions as long as either the solution is antisymmetric or inertial effects are ignored.

When $s \neq 0$ or $q \neq 0$, the functions $f$ and $g$ are not symmetric, as shown in figures 6 and 7, where $s=0.1$, $q=0.01, W e=4, R e=0.4$. Since our numerical scheme is designed to start with $a=0$, and $a$ is incremented in small steps, and the solutions for $f$ and $g$ are obtained from iterating on the respective solutions for the previous value of $a$, the scheme converges to a smooth solution while $a$ remains small. For example, in figures 6 and 7, we find that the solutions are smooth until $a$ is slightly over 0.8 . A critical value of $a$ is then reached at which the discriminant is zero, and the scheme has convergence problems. The solutions plotted for $a \leqq 0.8$ can be reproduced with finer meshes. Above the critical value of $a$, the Newton scheme again 


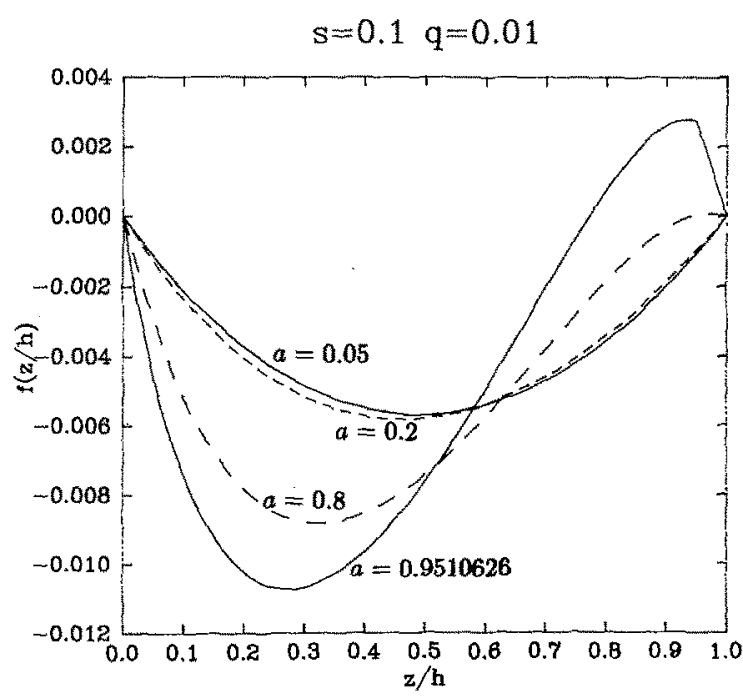

Fig. 6. Graph of $f(z / h)$ versus $z / h, s=0.1, q=0.01$.

$\lambda=1, C=1, \varrho=0.1, h=1, \Omega=4, W e=4, R e=0.4$, zeroshear-rate viscosity $=1$

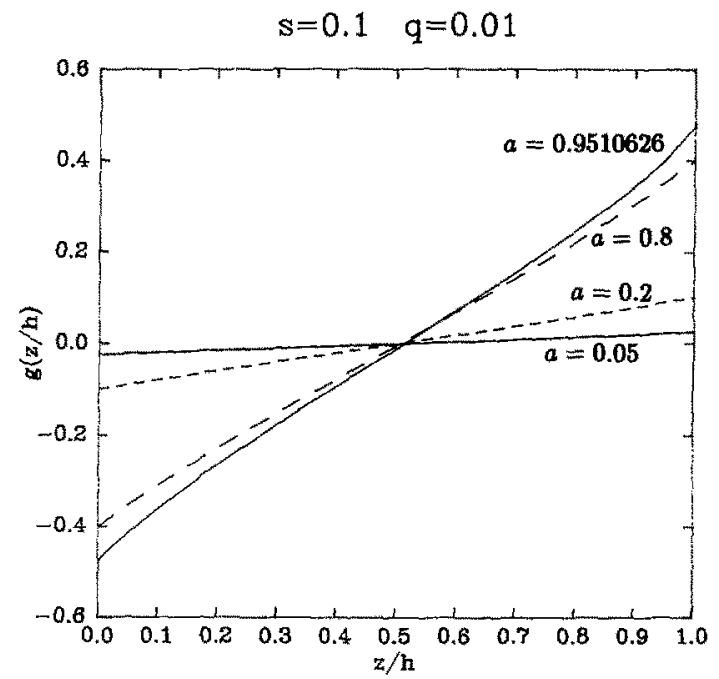

Fig. 7. Graph of $g(z / h)$ versus $z / h, s=0.1, q=0.01$.

$\lambda=1, C=1, \varrho=0.1, h=1, \Omega=4, W e=4, R e=0.4$, zeroshear-rate viscosity $=1$

converges but the solutions are non-unique and can be non-smooth with discontinuous derivatives, with the discrimimant being negative on part of the solution. Which solution the Newton scheme chooses to converge to depends on the number of meshpoints and may be influenced by round-off errors as above. Using twenty meshpoints, the scheme converged at $a=$ 0.9510626 to the non-smooth solutions displayed in figures 6 and 7 . The graph of this solution has been plotted with interpolation from the solution computed with twenty meshpoints up to $z / h=0.95$. The kink in

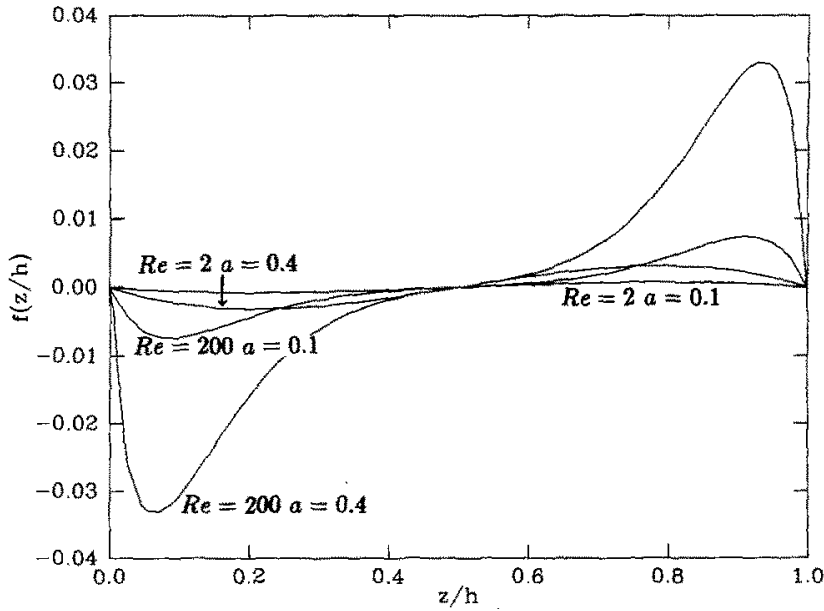

Fig. 8. Graph of $f(z / h)$ versus $z / h, s=q=0$. $\lambda=0.5, C=2.5, h=2, \Omega=0.5, W e=1$, zero-shear-rate viscosity $=10$

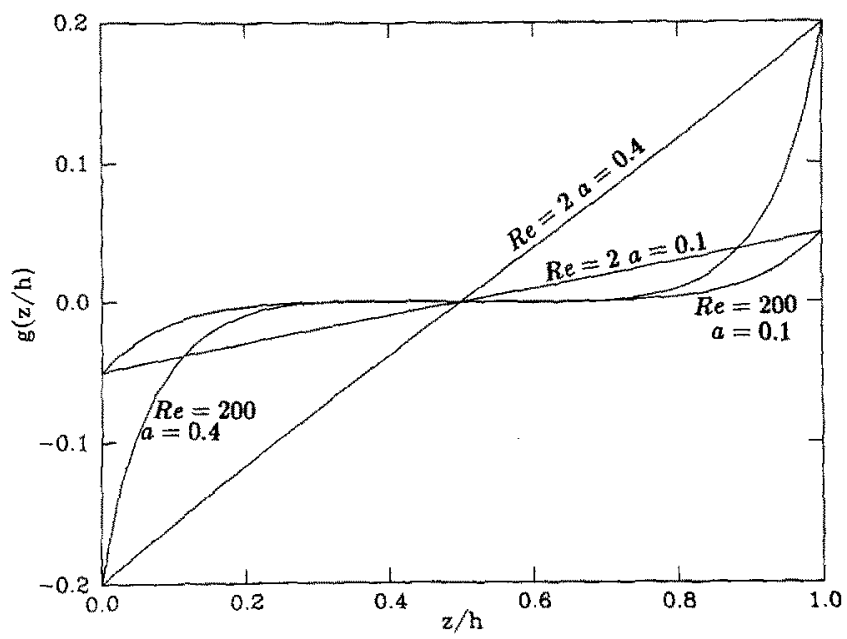

Fig. 9. Graph of $g(z / h)$ versus $z / h, s=q=0$. $\hat{\lambda}=0.5, C=2.5, h=2, \Omega=0.5, W_{e}=1$, zero-shear-rate viscosity $=10$

the solution occurred at the meshpoint next to the boundary. This is not surprising, since $x$ assumes its largest values at the boundary (cf. the lemma in section 3 ), and hence $D$ would first become zero at the boundary.

The appearance of discontinuous solutions may be related to local yielding, which is observed experimentally (cf. Wissbrun [20]). We note that although the flow under study is not viscometric, there is a qualitative similarity with simple shear flow. The Currie model has a shear stress maximum in shear flow, and if shear rates exceed a critical value, then discontinuous solutions are also possible. In the geometry studied in this paper, solutions with discontinuous derivatives 
were shown to exist for nonlinear elastic materials with non-convex stored energy functions (cf. Rajagopal and Wineman [21]).

If the Weissenberg number is small and the Reynolds number is large, there is a boundary layer structure to the solution as is to be expected from the study of simpler models [13], [14], see also Joseph [22], where the case of large $\Omega$ and small $a / h$ is considered for arbitrary simple fluids Examples are shown in figures 8 and 9.

\section{Acknowledgements}

The work of K. R. Rajagopal was supported by the National Science Foundation under the Solid Mechanics and Dynamical Systems programs. Part of the research was undertaken while K. R. Rajagopal was visiting the Mathematics Research Center at the University of Wisconsin. The research of M. and Y. Renardy was sponsored by the United States Army under Contract No. DAAG29-80-0041. M. Renardy was also supported by the National Science Foundation under Grants No. MCS-8215064 and DMS-8451761.

\section{References}

1. Maxwell B, Chartoff RP (1965) Trans Soc Rheol 9:41-52

2. Blyler LL, Kurtz SJ (1967) J Appl Poly Sci 11:127-131

3. Bird RB, Harris EK (1968) AIChE J 14:758-761

4. Huilgol RR (1969) Trans Soc Rheol 13:513-526

5. Kearsley EA (1970) J Res Nat Bur Stand 74 c: $19-20$

6. Gordon RJ, Schowalter WR (1970) AIChE J 16:318-320

7. Abbott TN, Walters K (1970) J Fluid Mech 40:205-213

8. Goldstein C, Schowalter WR (1975) Trans Soc Rheology 19:1-19

9. Rajagopal KR (1982) Arch Rat Mech Anal 79:39-47
10. Coleman BD (1962) Trans Soc Rheol 6:293-300

11. Noll W (1962) Arch Rat Mech Anal 11:97-105

12. Goddard JD (1983) Quart Appl Math 41:107-118

13. Rajagopal KR, Gupta AS (1981) Int J Eng Sci 19:14011409

14. Rajagopal KR, Wineman AS (1983) J Rheology 27:509515

15. Wang CC (1965) Arch Rat Mech Anal 20:329-340

16. Rivlin RS, Ericksen JL (1955) J Rat Mech Anal 4:323-425

17. Bernstein B, Kearsley EA, Zapas LJ (1963) Trans Soc Rheol 7:391-410

18. Currie PK (1982) J Non-Newtonian Fluid Mech 11:53-68

19. Doi M, Edwards SF (1978) J Chem Soc Faraday 74:17891832; (1979) $75: 38-54$

20. Wissbrun $\mathrm{KF}$, private communication

21. Rajagopal KR, Wineman AS (1984) J Structural Mech $12: 471-482$

22. Joseph DD (1977) Arch Rat Mech Anal 66:311-344

(Received September 27, 1985)

Authors' addresses:

Dr. K. R. Rajagopal

Department of Mechanical Engineering

University of Pittsburgh

Pittsburgh, PA 15261 (U.S.A.)

Dr. M. Renardy, (Mrs.) Dr. Y. Renardy

Department of Mathematics

Virginia Polytechnic Institute

and State University

Blacksburg, VA 24061 (U.S.A.)

Dr. A. S. Wineman

Department of Mechanical Engineering

and Applied Mechanics

University of Michigan

Ann Arbor, MI 48109 (U.S.A.) 